\title{
Localization of Surface States in Disordered Step Lattices
}

\author{
F. Baumberger, ${ }^{1, *}$ M. Hengsberger, ${ }^{1}$ M. Muntwiler, ${ }^{1}$ M. Shi, ${ }^{2}$ J. Krempasky, ${ }^{2}$ L. Patthey, ${ }^{2}$ J. Osterwalder,,${ }^{1}$ and T. Greber ${ }^{1}$ \\ ${ }^{1}$ Physikinstitut der Universität Zürich, Winterthurerstrasse 190, CH-8057 Zürich, Switzerland \\ ${ }^{2}$ Swiss Light Source, Paul Scherrer Institut, CH-5232 Villigen, Switzerland \\ (Received 12 December 2003; published 13 May 2004)
}

\begin{abstract}
The character of the surface state wave function on regularly stepped $\mathrm{Cu}(111)$ is reinvestigated. It is shown that the qualitative change at terrace lengths around $17 \AA$ observed previously by Ortega et al. [Phys. Rev. Lett. 84, 6110 (2000)] must necessarily be described as a change from a propagating superlattice state to a terrace-confined quasi-one-dimensional state. This reconciles previous, apparently contradictory experimental results and sheds new light on the behavior of nearly free electrons in nanostructures. Possible mechanisms driving the localization are discussed on the basis of the surface state bulk penetration depth, which has been measured in both regimes.
\end{abstract}

DOI: 10.1103/PhysRevLett.92.196805

PACS numbers: 73.20.At, 79.60.Ht

The modification of electronic properties in nanoscale structures is an issue of both fundamental and technological importance. As artificial structures are miniaturized to dimensions below the electronic coherence length, interfaces can no longer be neglected, and quantum size effects become important. Prominent examples include semiconductor heterostructures, metallic quantum-well states in thin films, or quantized electron levels in clusters [1-3]. Shockley surface states form nearly free quasitwo-dimensional electron gases on noble metal (111) surfaces and have played a pivotal role in the exploration of quantum size effects on metal surfaces. Their low inelastic scattering rates and Fermi wavelengths considerably larger than interatomic distances have enabled detailed studies of the modification of the wave functions in various artificial structures, such as corrals of adatoms [4], single and pairs of monatomic steps [5-7], as well as periodic arrays of steps [8-16]. On the other hand, their charge density proved to be high enough to influence the energy balance of the surface and produce a feedback on the atomic arrangement $[13,17,18]$.

Although much of the physics of surface states interacting with lateral nanostructures may be described by elementary wave mechanics [7], there remain intriguing issues. In a recent paper, Ortega et al. reported a qualitative change in the surface state wave function on regularly stepped $\mathrm{Cu}$ surfaces when the average terrace length exceeded $\approx 17 \AA$ [11]. This crossover was interpreted as a "switch from step to terrace modulation" of the wave function, thereby resolving an apparent inconsistency between scanning tunneling microscopy data from large terraces, showing wave functions in the terrace plane, and photoemission data from vicinal surfaces, finding a Bloch wave vector in the optical surface plane [9]. However, photoemission studies on vicinal $\mathrm{Cu}$ always showed states with continuous parabolic dispersion, which has been taken as evidence for propagating states [9-11], while STM experiments found standing waves, confined on single terraces $[4,6]$. More recently, photoemission ex- periments on vicinal Au exhibited a crossover from propagating $2 \mathrm{D}$ states to confined $1 \mathrm{D}$ states, as the terrace width was increased [14].

Here, we report an extensive photoemission study from two vicinal $\mathrm{Cu}$ surfaces with terrace lengths of 16.3 and $25.2 \AA$. We confirm a qualitative change of the wave function and show that it is described by the crossover from a superlattice state on surfaces with terraces narrower than $\approx 20 \AA$ to $1 \mathrm{D}$ localized states on wider terraces. This resolves the prior inconsistency between STM and photoemission data, and reconciles the apparent incongruity between $\mathrm{Cu}$ and the other noble metals.

Photoemission experiments have been performed at the SIS beamline of the Swiss Light Source using a Scienta 2002 spectrometer. All data were taken at room temperature. The combined energy resolution (photons and electrons) ranged from $\approx 15 \mathrm{meV}$ at $h \nu=21 \mathrm{eV}$ to $\approx 50 \mathrm{meV}$ at $110 \mathrm{eV}$, and the angular resolution for all measurements was better than $\pm 0.15^{\circ}$. The two investigated samples, $\mathrm{Cu}(443)$ and $\mathrm{Cu}(665)$ are $B$-type vicinals of $\mathrm{Cu}(111)$. Their nominal structure consists of monatomic steps with (11) microfacets, separated by fcc(111) terraces of $L=7 \frac{1}{3}(16.3 \AA)$ and $11 \frac{1}{3}(25.2 \AA)$ atomic rows width. Clean and well ordered surfaces were prepared by repeated sputter/anneal cycles [12]. Standard deviations of the terrace width distributions $\leq 0.31 \mathrm{~L}$ for $\mathrm{Cu}(443)$ and $\leq 0.36 L$ for $\mathrm{Cu}(665)$ were calculated from the LEED (low energy electron diffraction) spot profiles.

Figure 1 shows the photoemission intensity distribution from the Shockley type surface states on $\mathrm{Cu}(443)$ and $\mathrm{Cu}(665)$, measured with various photon energies. The wave vector $k_{x}$ runs normal to the steps in the "uphill" direction. By varying the photon energy, the surface state wave function is probed at different wave vectors $k_{z}$ normal to the surface. For the extended photon energy range in our experiment $(21-110 \mathrm{eV}), k_{z}$ ranges from $\approx 2.8$ to $\approx 5.5 \AA^{-1}$, thus covering almost one reciprocal lattice vector between $\Gamma_{111}$ and $\Gamma_{222}$. 


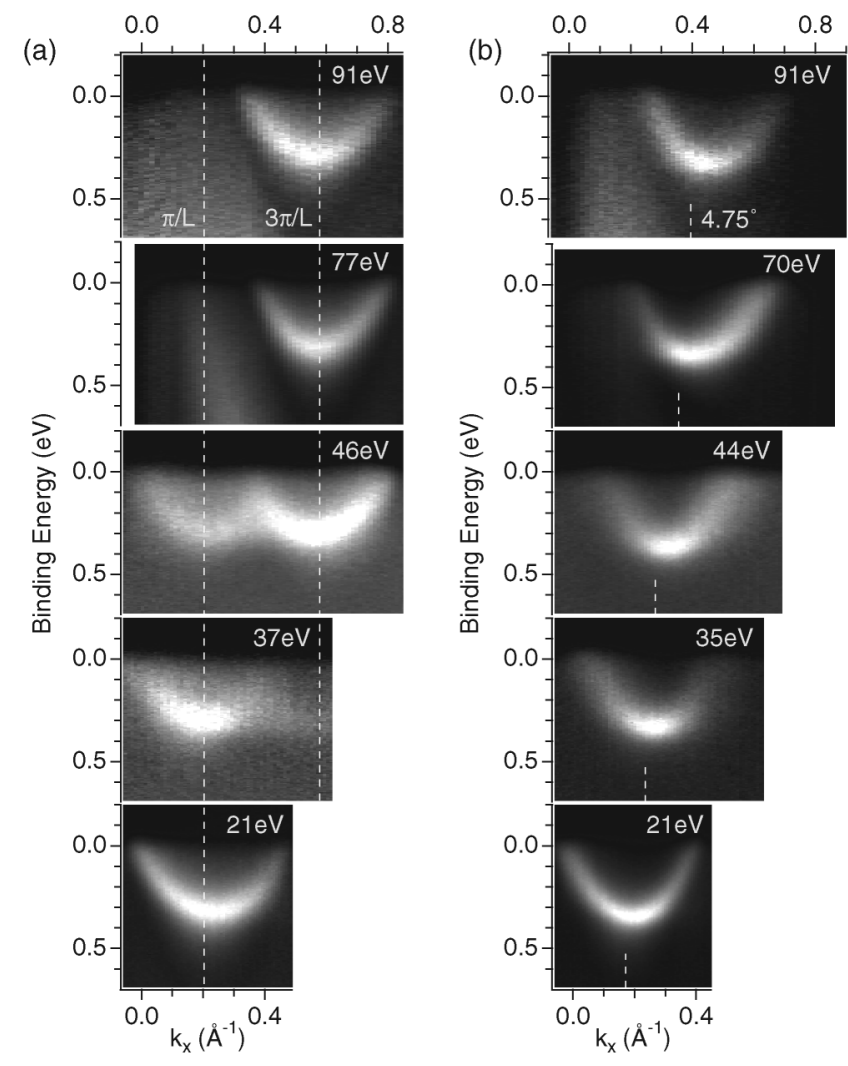

FIG. 1. Surface state dispersion perpendicular to the steps, measured with various photon energies, probing different wave vectors $k_{z}$ normal to the surface between $\mathbf{G}_{111}$ and $2 \mathbf{G}_{111}$. (a) $\mathrm{Cu}(443)$; (b) $\mathrm{Cu}(665)$.

Clearly, the two surfaces show a qualitatively different behavior. For $\mathrm{Cu}(443)$, the band bottom coincides for all photon energies with a zone boundary of the step superlattice [dashed lines in Fig. 1(a)]. This indicates a coherent interaction of the surface state wave function over several terraces, as confirmed recently by the observation of a band gap, opening at the Fermi level [13].

The main change between $\mathrm{Cu}(665)$ and $\mathrm{Cu}(443)$ is evident in the data sets around $45 \mathrm{eV}$. While the band is backfolded by a reciprocal step-lattice vector on $\mathrm{Cu}(443)$, there is no signature of the superlattice periodicity in the data from $\mathrm{Cu}(665)$. Instead the band bottom simply follows the $k_{x}$ values that correspond to the miscut angle of $4.75^{\circ}$, as observed previously by Ortega et al. at low photon energies [11]. The loss of any superlattice periodicity indicates a dramatic change of the initial state wave function. It obviously can no longer be described by a propagating superlattice state. However, its almost parabolic dispersion also appears to be incompatible with a 1D localized state, as observed by STM $[6,7,15]$ and photoemission [19] on large terraces of $\mathrm{Ag}(111)$ and $\mathrm{Au}(111)$.

In the following we show that the apparently parabolic dispersion of the Shockley surface state on $\mathrm{Cu}(665)$ does not imply freely propagating states. It is well described by the photoemission intensity from an incoherent ensemble of lateral quantum-well states, which arise due to the coherent reflection of the wave function at the step edges defining individual terraces $[6,7]$. The apparent dispersion turns out to be a consequence of the strong angular dependence of the matrix element, in conjunction with the spatial averaging in a photoemission experiment. This result will be demonstrated in adopting the experimental data with a model close to that of Mugarza et al. [19].

We start with the standard expression for the photocurrent $I(k, \omega)$ in the three step model:

$$
I(k, \omega) \propto\left|M_{f i}\right|^{2} A(k, \omega) f(\omega),
$$

where $A(k, \omega)$ is the single particle spectral function and $f(\omega)$ the Fermi-Dirac distribution. In order to calculate the matrix element, we neglect all components of the vector potential parallel to the surface plane, because the character of the surface state is largely dominated by $s p_{z}$ orbitals. For a plane wave final state $\Psi_{f}=e^{i \mathbf{k r}}$, the remaining integral is proportional to the Fourier transform of the initial state wave function along the $x$ coordinate. It can be solved analytically for the wave functions for a particle in a box $\Psi_{i_{x}} \propto \sin (N \pi x / L)$, where $N=1,2, \ldots$, is the quantum number of the state and $L$ the width of the well [14]:

$$
\left|M_{f i}\right|^{2} \propto \frac{N^{2}}{L^{3}} \frac{1-(-1)^{N} \cos \left(k_{x} L\right)}{\left[k_{x}^{2}-(\pi N / L)^{2}\right]^{2}} .
$$

The binding energy levels $\epsilon_{N}$ for states in a onedimensional quantum well are given by $\epsilon_{N}=$ $\epsilon_{0}-N^{2} \pi^{2} \hbar^{2} / 2 m^{*} L^{2}$, where $\epsilon_{0}$ is the "energy zero" for the surface state, which we expect to lie close to the band bottom $(0.39 \mathrm{eV})$ for the flat surface [20]. The atomic potentials of the terraces are taken into account by using the effective mass $m^{*} / m_{e}=0.41$ of the surface state on $\mathrm{Cu}(111)$. For weakly interacting systems, $A(k, \omega)$ reduces to a Lorentzian and the photocurrent, expected for a single, isolated quantum well, can be calculated from Eq. (1). The result is shown in Fig. 2(a), for the well width on $\mathrm{Cu}(665)$ of $25.2 \AA$. The first two states lead to the nondispersing levels at $\approx 0.35$ and $\approx-0.1 \mathrm{eV}$, which are both strongly modulated in intensity by the matrix element. Next, we extend the model to a distribution of decoupled quantum wells, by weighting $I(k, \omega)$ with the probability $P(L)$ to find a terrace of width $L$ [21]. Figure 2(b) shows a numerical integration of the weighted photocurrent, assuming a Gaussian $P(L)$ with a standard deviation $\sigma=0.36 L$, as obtained from the LEED analysis. The discrete energy levels seen in Fig. 2(a) are completely smeared out, and the intensity follows basically the free-electron-like dispersion with the same $m^{*}$ and $\epsilon_{0}$. For a meaningful comparison of experiment and simulation, the latter is superimposed on a constant background and multiplied by the Fermi function. The resulting image plot, shown in Fig. 2(c), closely resembles the experiment, reproduced in Fig. 2(d). The key features of the experimental line shapes may be seen in Fig. 2(e) and 2(f). In the center of the image, the energy distribution 

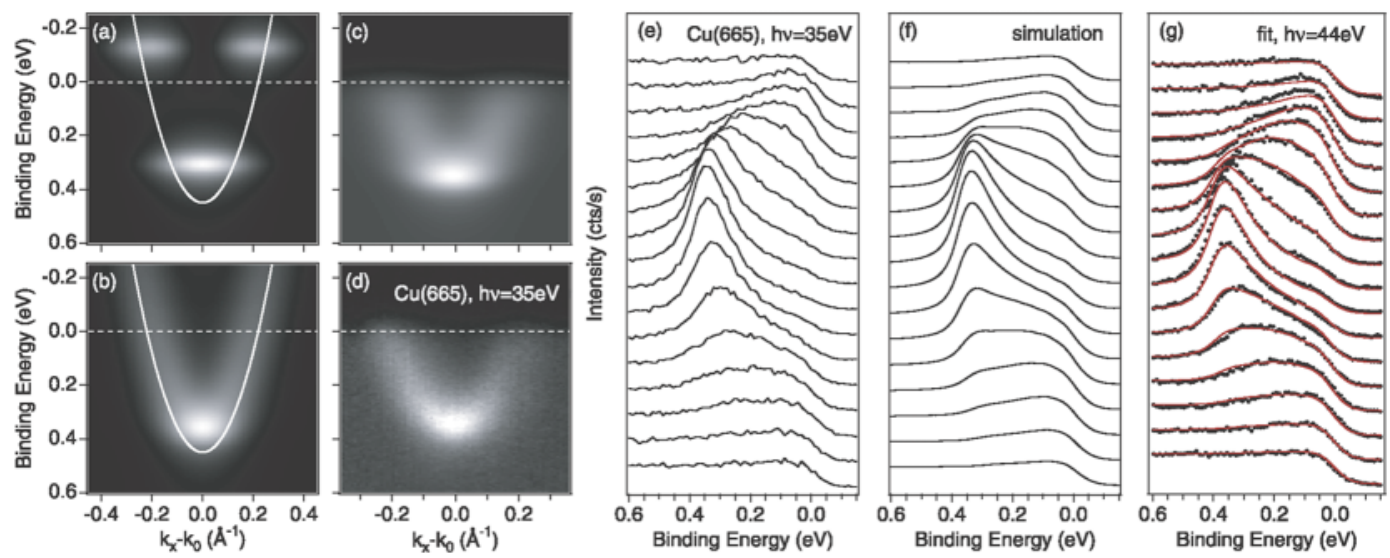

FIG. 2 (color online). Simulation of photoemission data. (a) Intensity distribution for isolated quantum wells of $25 \AA$ width, assuming a linewidth of $90 \mathrm{meV}$. (b) Same for a Gaussian distribution of the well width. (c) Simulated intensity distribution from (b) with a constant background and multiplied with a Fermi function. (d) Photoemission data from Cu(665). (e),(f) EDCs from (c) and (d). (g) Fit to a refined model (for details see text).

curves (EDCs) are intense and narrow but highly asymmetric. Upon approaching the Fermi level, the peaks become broader, and their asymmetry changes. All these features are well reproduced in the simulation. Note that the relative intensity of the subbands is fixed by Eq. (2). The remaining differences are mostly due to the oversimplified background and the photon energy dependent change in the intensity of left and right parabola branch. This is demonstrated by fitting a refined model to the data, which includes a background that accounts for the reduction of indirect transitions in the projected band gap, and an empirical parameter to better follow the peak intensities [Fig. 2(g)].

The qualitative change of the wave function is confirmed by the behavior of the surface state linewidths $\Gamma$, shown in Fig. 3. The value of $\Gamma / 2=45 \mathrm{meV}$ fitted to the $\mathrm{Cu}(665)$ data clearly deviates from the linear increase in step-density found for the superlattice states [23]. This points to an obvious difference between the two regimes. Since the superlattice state propagates along the macroscopic surface plane, it is not orthogonal to the bulk states and is thus in strict terms a resonance [12,24]. This allows the decay of the photohole by scattering with bulk states on terrace sites, with a decay-rate proportional to the miscut angle (which, for small angles, is proportional to the step-density) [12]. The quantum-well state, on the other hand, can interact with bulk states only at the step-edges, resulting in a longer lifetime.

It has been speculated $[12,14]$ that the surface statesurface resonance transition might increase the bulk penetration depth of the resonance, which could cause its reduced sensitivity to the step potentials, which in turn may trigger the interaction over multiple terraces. Conceptualizing this view, we might understand the localized state as more natural, while the propagating resonances result because the system tries to avoid the high confinement energy on narrow terraces. In order to test this hypothesis, we have evaluated the surface state cross section as a function of $k_{z}$ in both regimes (Fig. 4). The width of the roughly Lorentzian cross section resonance gives a measure for the bulk decay length $\beta$, as it essentially reflects the Fourier transform of the initial state wave function $\Psi_{i_{\tau}}=e^{(-\beta z)} \cos \left(k_{L} z+\delta\right)\left[k_{L}=\mathbf{G}_{111} / 2\right]$, broadened by a final state contribution [25]. Obviously, there is no difference within the experimental accuracy between the two regimes, and the half-width of the resonance $\left(0.67 \AA^{-1}\right)$ is very close to the value found for $\mathrm{Cu}(111)\left(0.71 \AA^{-1}\right)[26]$.

Explanations of the crossover, based on different effective step potentials felt by the quantum-well and superlattice states are thus likely inadequate. Instead, the surface state band structure energy provides an alternative grasp of the transition. Recently, we have demonstrated that the superlattice state gains significant energy due to the opening of a band gap at the Fermi level for terrace widths around $16 \AA$ [13]. A further increase in

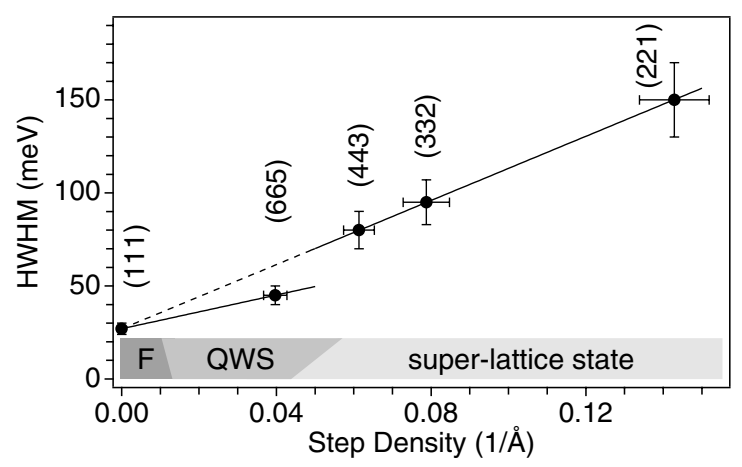

FIG. 3. Surface state linewidth as a function of step density [22]. Error bars reflect the scatter in different measurements. The three surface state phases [unperturbed (F), quantum-well states (QWS), and propagating superlattice state] are schematically indicated. Values in the superlattice regime have been obtained with comparable analyses and are published in Refs. [12,13]. 


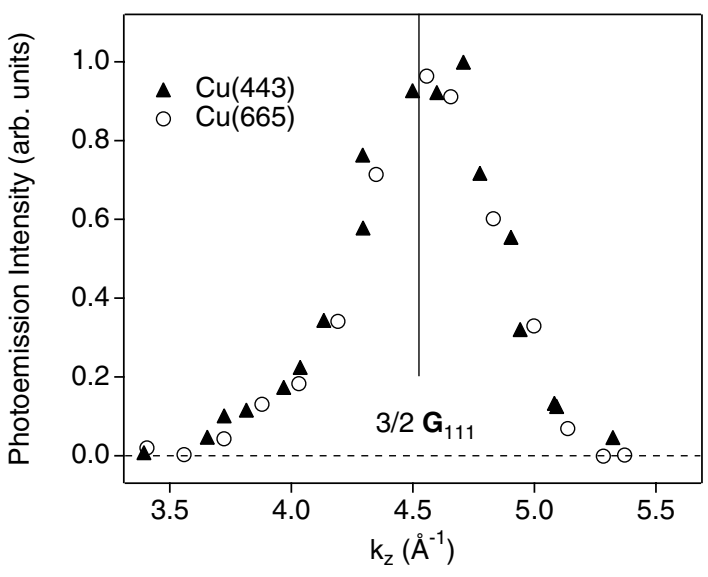

FIG. 4. Photoemission intensity as a function of perpendicular momentum $k_{z}$ for the lateral quantum well state on $\mathrm{Cu}(665)$ and the superlattice state on $\mathrm{Cu}(443)$. Data have been normalized to the photon flux.

terrace size causes the gap to move into the occupied states. As a consequence, the energy gain due to the gap opening is reduced and even vanishes for $L \gtrsim 25 \AA$.

Although energetic considerations point in the right direction, they might not be sufficient to explain the observed crossover. Ortega et al. proposed an onset of energy conserving scattering with bulk states as the projected band gap closes to explain the transition to "step modulation" on narrow terraces [11]. However, the geometrical coincidence between the onset of scattering with step-lattice vectors and the miscut angle, where the transition was observed, holds only for surface state electrons at the band bottom, and these states have no available final states to scatter into. Furthermore, the STM experiments of Bürgi et al. indicate that scattering with bulk states is important even for very large terrace widths [7]. This, together with the identical bulk penetration depth in both regimes, makes it unlikely that interaction with bulk electrons drives the transition.

While the total energy of the electronic surface state must be important for a complete understanding of this transition from extended superlattice states to localized quantum-well states, it is likely that fluctuations in the step-lattice promote localization. Evidence for the relative importance of energetics and disorder induced localization could come from electronic structure calculations and molecular dynamics simulations. On this note it would be interesting to reexamine the weak onedimensional surface state found in the superlattice regime [22].

In conclusion, we have demonstrated the localization of surface states on stepped $\mathrm{Cu}(111)$ surfaces with $L>$ $20 \AA$. With this finding, a consistent picture of surface state confinement on stepped noble metal surfaces emerges, which is of direct relevance for an understanding of a variety of processes they govern, including adatom diffusion, molecular ordering or surface reconstructions
$[17,18]$. On very large terraces, surface states propagate free electron like. As the step-step separation shrinks to below the coherence length, localized states with a discrete energy spectrum form and for even smaller terrace widths ( $\ 20 \AA$ ), the states propagate again with the steps now acting as a superlattice.

We thank N. J. C. Ingle, A. Kara, and K. M. Shen for fruitful discussions and the Swiss National Science Foundation for financial support. The experiments have been performed at the Swiss Light Source, Paul Scherrer Institute, Villigen, Switzerland.

*Present address: Department of Applied Physics, Stanford University, Stanford, California 94305, USA. Electronic address: baumberger@stanford.edu

[1] C.W. J. Beenakker and H. Vanhouten, Solid State Phys. Adv. Res. Appl. 44, 1 (1991).

[2] M. Valden, X. Lai, and D.W. Goodman, Science 281, 1647 (1998).

[3] M. N. Baibich et al., Phys. Rev. Lett. 61, 2472 (1988).

[4] E. J. Heller et al., Nature (London) 369, 464 (1994).

[5] M. F. Crommie, C. P. Lutz, and D. M. Eigler, Nature (London) 363, 524 (1993).

[6] P. Avouris and I.W. Lyo, Science 264, 942 (1994).

[7] L. Bürgi et al., Phys. Rev. Lett. 81, 5370 (1998).

[8] R. S. Williams et al., Phys. Rev. Lett. 41, 323 (1978).

[9] A. P. Shapiro, T. Miller, and T.-C. Chiang, Phys. Rev. B 38, 1779 (1988).

[10] O. Sánchez et al., Phys. Rev. B 52, 7894 (1995).

[11] J. E. Ortega et al., Phys. Rev. Lett. 84, 6110 (2000).

[12] F. Baumberger, T. Greber, and J. Osterwalder, Phys. Rev. B 64, 195411 (2001).

[13] F. Baumberger et al., Phys. Rev. Lett. 92, 016803 (2004).

[14] J. E. Ortega et al., Phys. Rev. B 65, 165413 (2002).

[15] K. Morgenstern, K. F. Braun, and K. H. Rieder, Phys. Rev. Lett. 89, 226801 (2002).

[16] M. Hansmann et al., Phys. Rev. B 67, 121409(R) (2003).

[17] S. Lukas, G. Witte, and Ch. Wöll, Phys. Rev. Lett. 88, 028301 (2002).

[18] J. Repp et al., Phys. Rev. Lett. 85, 2981 (2000); N. Knorr et al., Phys. Rev. B 65, 115420 (2002).

[19] A. Mugarza et al., Phys. Rev. Lett. 87, 107601 (2001).

[20] A possible penetration of $\Psi_{i}$ into the steps is treated by allowing $\epsilon_{0}$ to be slightly higher than $0.39 \mathrm{eV}$.

[21] A. Beckmann et al., Surf. Sci. 375, L363 (1997).

[22] F. Baumberger, T. Greber, and J. Osterwalder, Phys. Rev. B 62, 15431 (2000); X. J. Shen et al., ibid. 63, 165403 (2001).

[23] All linewidths throughout this Letter refer to the band bottom. The line shape at higher energies is completely dominated by inhomogeneous broadening due to the averaging over the terrace width distribution.

[24] R. Matzdorf and A. Goldmann, Surf. Sci. 400, 329 (1998).

[25] S. G. Louie et al., Phys. Rev. Lett. 44, 549 (1980).

[26] S. D. Kevan and R. H. Gaylord, Phys. Rev. Lett. 57, 2975 (1986). 